\title{
P01-9-26 Poster session
}

\section{Anti-helicobacter pylori, inflammatory effects and constituent analysis of Modified Xiaochaihutang for the treatment of chronic gastritis and gastric ulcer}

\author{
Yanwen Liu, Lijuan Hu, Huanhuan Wu, Xin Chen
}

Hubei University of Chinese Medicine, China

Background: Modified Xiaochaihutang (MXCHT) prescription was investigated to confirm the anti-helicobacter pylori (H. Pylori) and anti-inflammatory effects, the main constituents of different extract for elaborating pharmacodynamic material basis, and providing a scientific basis for the treatment of gastritis clinically.

Methods: The activity of full prescription and different extract towards H. pylori was assessed by agar diffusion method in vitro, and the minimal inhibitory concentration (MIC) was also determined by fluid dilution method. The activity of full prescription and different extract towards inflammatory were assessed by mouse ear edema model and rat paw edema model. Active constituents in plasma after oral administration of MXCHT were revealed by HPLC-TOF-MS/MS.

Results: For results, the bacterial inhibition diameter and MIC for full prescription and 4 extracts were dramatically decreased compared with control group. Xylene-induced mouse ear edema model, high and low dose group of the full prescription and $\mathrm{n}-\mathrm{BuOH}$ extract, high dose group of water extract, and EtOAc extract shown significantly inhibition. Carrageenan-induced rat paw edema model, high and low dose group of the full prescription and $\mathrm{n}-\mathrm{BuOH}$ extract, high dose group of water extract, and EtOAc extract could also significantly reduce paw swelling of rats. 4 prototype components and 4 metabolites were identified after oral administration of EtOAc extract. In addition, 6 prototype components and 6 metabolites were identified in $\mathrm{n}-\mathrm{BuOH}$ extract.

Conclusions: The present results reveal that EtOAc extract and $\mathrm{n}-\mathrm{BuOH}$ extract were effective extract for anti-H. Pylori and anti-inflammatory in MXCHT, and also provide experiment data for the clinical treatment of chronic gastritis. 